\title{
A Mathematical Model of the Error of Measuring Instruments for Investigating the Dynamic Characteristics
}

\author{
Dimitar Dichev ${ }^{1, *}$, Fotini Kogia ${ }^{1,2}$, Hristiyana Nikolova ${ }^{3}$ and Dimitar Diakov ${ }^{3}$ \\ ${ }^{1}$ Technical University-Gabrovo, 5300 Gabrovo, 4 H. Dimitar Str. \\ ${ }^{2}$ Department of Electrical Engineering, Eastern Macedonia and Thrace Institute of Technology, St. Lucas, 65404, Kavala, Greece \\ ${ }^{3}$ Technical University-Sofia, 1000 Sofia, 8 Kl. Ohridski Blvd.
}

Received 5 June 2018; Accepted 14 December 2018

\begin{abstract}
The present paper views the results related to the solution of a complicated problem in the area of measuring equipment. The proposed mathematical model makes possible the transfer of the measuring unit, the calibration and verification of measuring equipment operating in dynamic mode on the basis of an output measuring instrument calibrated in static mode. The measuring equipment under study is a stand-simulator based on a six-degree-of-freedom Stewart Platform for verifying and calibrating instruments measuring the parameters of a ship's motion. The mathematical model is developed on the basis of the direct kinematics problem of parallel mechanisms.
\end{abstract}

Keywords: metrology, Stewart platform, kinematics, stand-simulator, calibration

\section{Introduction}

The metrological theory related to dynamic measurement aims to achieve traceability at the accuracy level required by today's science. From this perspective dynamic measurements are distinguished for a number of specific features related to static measurements. For example, the investigation of the accuracy of instruments operating in dynamic mode is distinguished for the fact that it is necessary to define the error of the reading of not only the measured quantity but also of time $[1,2]$. The methods for measuring time are known and accurate enough. Of course, the error component generated upon time fixing should be taken into consideration. The problem related to the definition of the reading error of the measured quantity is more complicated. If the measuring instrument allows measurement in static mode, then its error can be determined on the basis of the respective system of references ensuring the quantity in static mode. If the measuring devices cannot be graduated in static mode, it is necessary to develop the respective references and hierarchical diagrams. The output measuring instruments in those hierarchical diagrams should be calibrated with the help of the existing references in static mode $[3,4,5,6]$. The solution of the problem on the basis of this approach has a number of difficulties mainly caused by the need for harmonizing the characteristics of the output measuring instruments to the dynamics of the quantity measured. Therefore, an appropriate mathematical model should be elaborated.

The nature of the problem related to the provision of the required accuracy to the stand equipment for calibrating and verifying the measuring instruments and systems that determine the parameters of a ship's motion can be viewed

*E-mail address: dichevd@abv.bg

ISSN: $1791-2377$ @ 2018 Eastern Macedonia and Thrace Institute of Technology. All rights reserved.

doi:10.25103/jestr.116.03 in this sense. The purpose of the equipment is to reproduce in a reference format the ship's motion with respect to its six degrees of freedom. On the ground of the characteristics of a ship's motion with respect to its six degrees of freedom which must be reproduced by the equipment, we can specify that the most apt variant of its development is using parallel mechanisms (a hexapod of six degrees of freedom). The development of the equipment is considered in details in [7].

To investigate the equipment accuracy and to develop a hierarchical diagram is a complicated task that requires the elaboration of an adequate mathematical model providing the respective procedures, which is actually the aim of the present paper.

\section{An analysis of the elements that build up the accuracy estimation system}

To achieve the above aim it is necessary to analyze the structure of the measuring equipment under estimation. This equipment is described in details in [7]. Its mechanical module is a hexapod which is actually a closed kinematic chain between the stationary base 1 and the motion platform 2 having six degrees of freedom (fig.1). Unlike conventional manipulators, the ones based on the hexapod perceive loading as space trusses. The six degrees of freedom are provided by the six actuators 3 , each of which consists of two elements and an active translational kinematic pair. The motion of the platform according to the respective law is guided by the current assignments of each actuator. Consequently the reference quantity defined in this case as a motion of the platform with respect to each degree of freedom is a function of the actual change of the lengths of the actuators according to the time coordinate. This principle is the core of the proposed accuracy estimation system of the measuring equipment because it relates the error $\Delta L_{i}$ of the 
current values of the actuator length to the accuracy upon reproducing the set motion of the platform.

The error $\Delta L_{i}$ is determined by the difference

$$
\Delta L_{i}=L_{i}^{d}-L_{i}^{u}, i=1,2, \ldots, 6
$$

where $L_{i}^{d}$ is the actual value of the length of the $i$-th actuator, $L_{i}^{u}$ - the current assignment of the $i$-th actuator.

Formula (1) defines error $\Delta L_{i}$ in static mode. Actually this error is dynamic because it is formed by the dynamically changing quantities, i.e.

$$
\Delta L_{i}(t)=L_{i}^{d}(t)-L_{i}^{u}(t), i=1,2, \ldots, 6 .
$$

It is necessary to mention that the values of error $\Delta L_{i}$, obtained according to formula (1) and (2), are close. However, formula (1) provides a little bit higher values with a difference not greater than $10 \%$.

To determine the values of error $\Delta L_{i}$, it is required to develop a measuring instrument which will function as an output device within the hierarchical diagram. The block diagram of the latter is shown in fig. 2 and the constructive model developed in SolidWorks is shown in fig. 3. It is developed as a stand measuring system which consists of a common base platform where a system for measuring the lengths of the actuators and a base holder are mounted. When mounting the actuator under verification 2 onto the base holder 3, its physical orientation and centring with respect to the coordinate axes of the base 1 are implemented (fig. 3). The carriage 4 moving along the cylindrical guides is attached to the movable element of the actuator. The sensor of the measuring system 5 is mounted on the carriage, which makes possible to measure the current length of the actuator. Measuring information is transferred by means of appropriate interface for connecting the measuring system to a computer where after processing in compliance with (1) or (2) the sought values of error $\Delta L_{i}$ are obtained (fig.2). The signals controlling the actuator are fed by the computer interface.

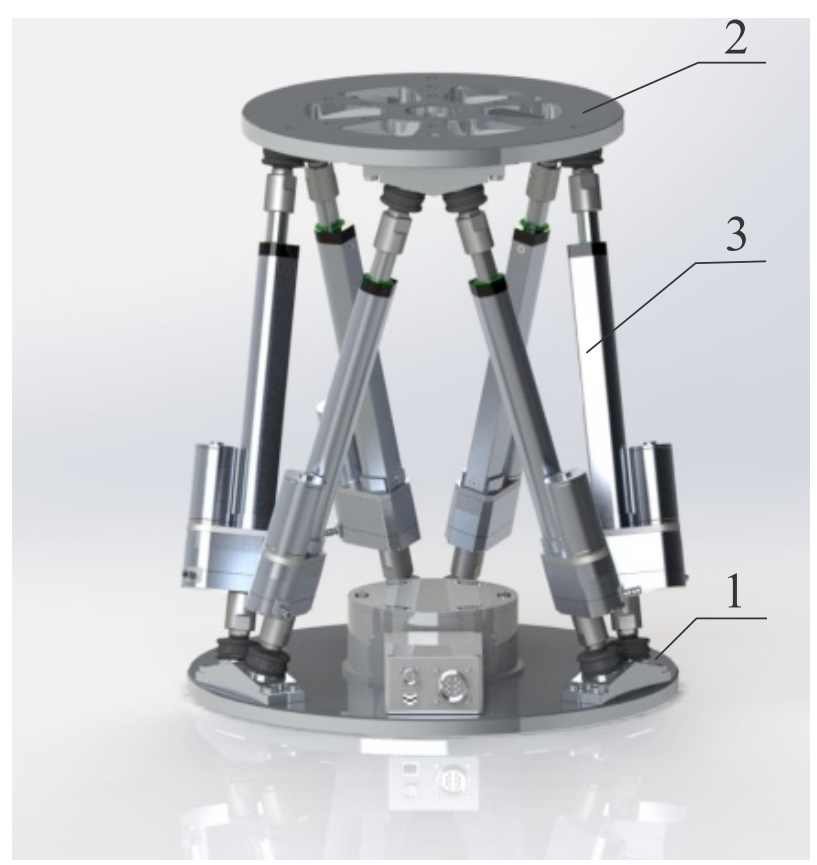

Fig. 1. Model of the estimated measuring instruments
The design of the equipment is relatively simple, which reduces the influence of the instrumental errors. A distinguishing feature of the proposed stand measuring system is that the transfer of the measuring unit from the referent elements could be done in static mode through direct methods. The same is true for calibration and verification.

The measuring information obtained for error $\Delta L_{i}$ makes possible to develop a procedure for calibration or estimation of the hexapod error. This procedure is based on the mathematical model defining the accuracy characteristics of the measuring equipment in a function of $\Delta L_{i}$.

\section{A mathematical model for determining the measurement equipment error}

The mathematical model is based on the kinematic problems of parallel mechanisms since the mechanical module of the equipment is built in the form of a hexapod. The kinematic problem provides a solution in relation to the dependences of the output coordinates of the motion platform on the lengths of the actuators. The problem can be divided into two parts a forward problem and an inverse problem. The purpose of the inverse problem is to determine the change of the lengths when the output coordinates are set, which actually coincides with the problem related to the control of the actuators linked to the motion along the set trajectory of the motion platform. The forward problem aims to define the output coordinates when the lengths are known. The essence of this problem coincides with the above concept for determining the accuracy upon reproducing the set motion of the operating platform depending on errors $\Delta L_{i}$ of the current values of the actuators lengths.

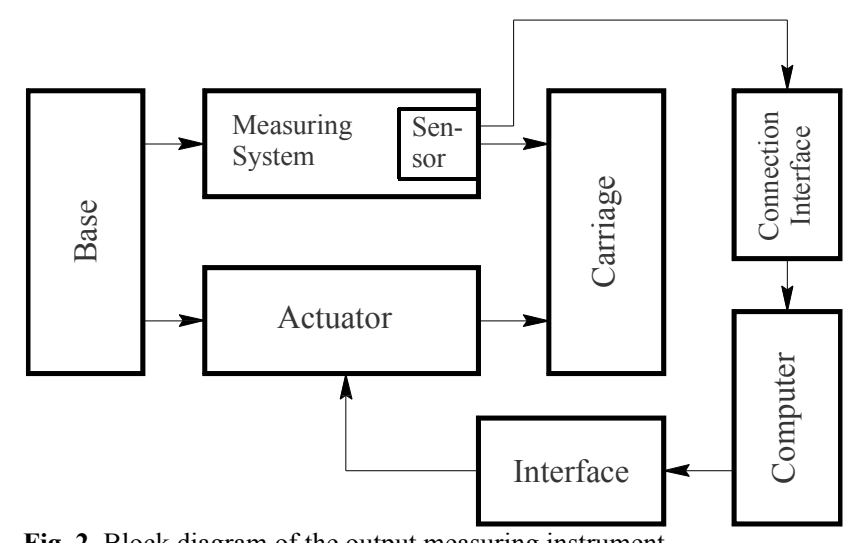

Fig. 2. Block diagram of the output measuring instrument

One of the first studies where the inverse problem is formulated and solved as completely as possible is [8]. It has a unique and very simple solution. Unlike it the solution of the forward kinematic problem is very complicated and results in a number of different configurations of the operating platform for the set lengths of the actuators. In this respect in the late 1980s and early 1990s its solution played a key role in the theory studying the Stewart platform. Today, as it is stated in $[9,10]$ for example, the kinematic problem is solved for each possible configuration of the platform. However, some indirect problems remain unsolved. In particular, the problem in relation to finding a unique solution of the forward problem among the set of possible solutions defining the configuration of the platform $[11,12]$. 


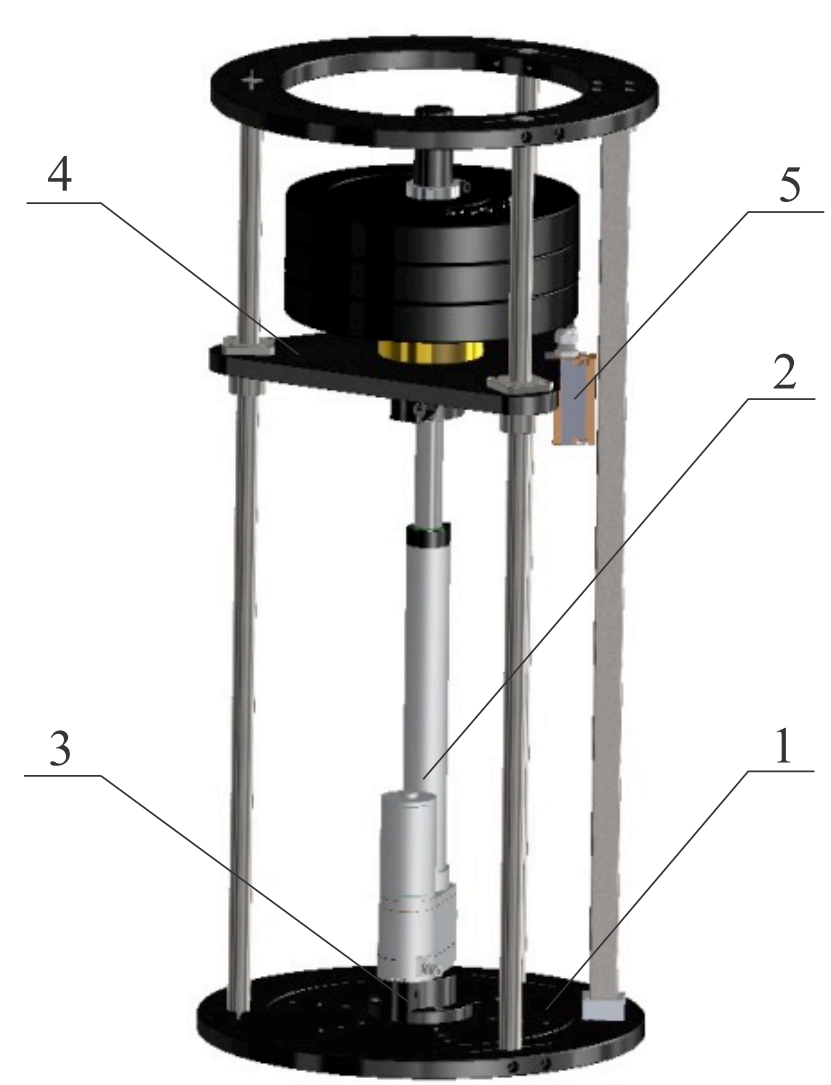

Fig. 3. Constructive model of the referent measuring instrument

The configuration space of the 6-degree-of-freedom Stewart platform is determined by the conditions of the closed-loop vector. The mathematical model is derived on the basis of the vector-projection equations defined by the closed kinematic chain shown in the kinematic diagram in fig. 4. It has the form [13]

$$
\vec{P}+\vec{r}_{i}-\vec{R}_{i}-\vec{L}_{i}=0
$$

where $\vec{P}=\overrightarrow{O_{B} O_{P}}$ is the radius-vector to the beginning of the mobile coordinate system $O_{P} x y z$.

In the kinematic diagram it is assumed that the actuators are weightless rods denoted by $B_{i} P_{i}, i=1,2, \ldots, 6$. Rods $B_{i} P_{i}$ consist of two elements connected by a translational kinematic pair. Points $P_{i}$ lie on the operating platform (fig.4). They are placed on a circle of a radius $r$ and a centre $O_{P}$, which coincides with the centre of mass of the operating platform. Points $B_{i}$, lying on a circle of a radius $R$ and a centre $O_{B}$, are placed on the base platform. The first element of each rod is connected to the base platform by a joint. There is a possibility for its angular displacement towards the base. The translational kinematic pair provides linear motion to the second element in the direction defined by the unit vectors $e_{i}$ connected to the respective rods $B_{i} P_{i}$.

The immobile coordinate system $O_{B} X Y Z$ is connected to the base. Its center coincides with point $O_{B}$ and axis $O_{B} Z$ is directed along the normal. The mobile coordinate system $O_{P} x y z$ is connected to the operating platform. The distance between point $O_{B}$ and point $O_{P}$ in initial position is $H_{0}$.

The following radius vectors defining the kinematics of the system are denoted in the kinematic model in fig. 4:

- $\vec{R}_{i}$ - a radius vector to points $B_{i}$, presented in the absolute coordinate system $O_{B} X Y Z$;

- $\vec{r}_{i}$ - a radius vector to points $P_{i}$ of the platform, set in the immobile coordinate system $O_{B} X Y Z$;

- $\vec{e}_{i}$ - a unit radius vector connected to the actuators, $\left|\vec{e}_{i}\right|=1$;

- $\vec{L}_{i}$ - a radius vector of the i-th actuator, $\vec{L}_{i}=\vec{B}_{i} P_{i}=L_{i} \vec{e}_{i} ; i=1,2, \ldots, 6$.

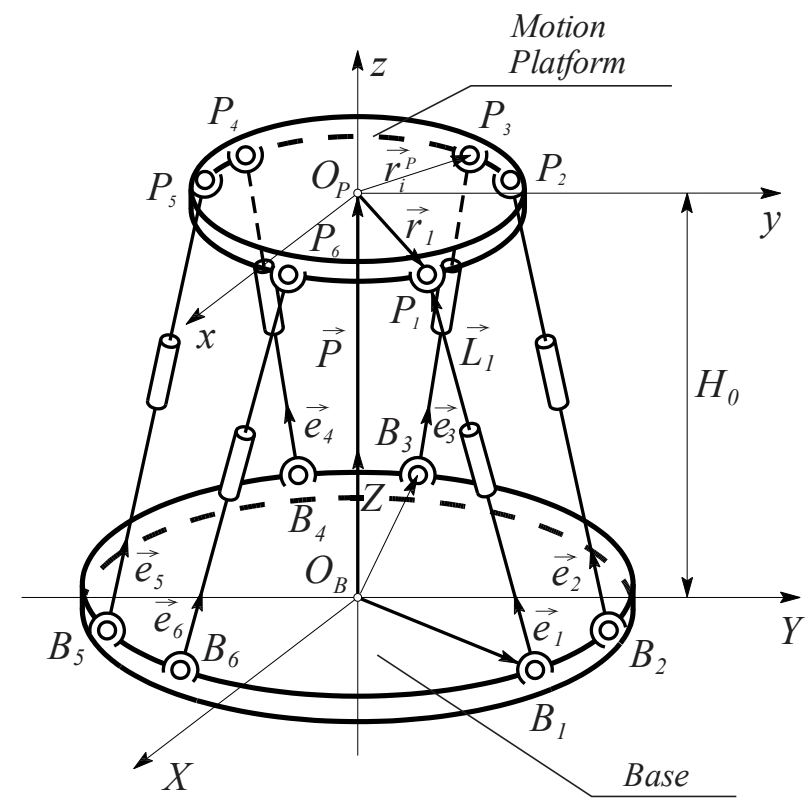

Fig. 4. Kinematic chain of the hexapod

The position of the platform in the immobile coordinate system is determined by a 6-dimensional column vector of the type

$\vec{R}_{P}=\left[\begin{array}{llllll}X_{P} & Y_{P} & Z_{P} & \alpha & \beta & \gamma\end{array}\right]^{T}$.

The three-dimensional column vector $\vec{r}_{i}$ can be presented by the vector relationship

$\vec{r}_{i}=[T] \cdot \vec{r}_{i}^{P}$

where $\vec{r}_{i}^{P}$ is a three-dimensional column vector of the coordinates of the platform spherical joints; $[T]$ is a square $3 \times 3$ matrix transforming the orientation of the mobile coordinate system $O_{P} x y z$ with respect to the immobile one $O_{B} X Y Z$, and it is of the type

$$
[T]=\left[\begin{array}{ccc}
\mathrm{c} \alpha \cdot \mathrm{c} \beta & \mathrm{c} \beta \cdot \mathrm{s} \beta \cdot \mathrm{s} \gamma-\mathrm{s} \alpha \cdot \mathrm{c} \gamma & \mathrm{c} \alpha \cdot \mathrm{s} \beta \cdot \mathrm{c} \gamma+\mathrm{s} \alpha \cdot \mathrm{s} \gamma \\
\mathrm{s} \alpha \cdot \mathrm{c} \beta & \mathrm{s} \alpha \cdot \mathrm{s} \beta \cdot \mathrm{s} \gamma+\mathrm{c} \alpha \cdot \mathrm{c} \gamma & \mathrm{s} \alpha \cdot \mathrm{s} \beta \cdot \mathrm{c} \gamma-\mathrm{c} \alpha \cdot \mathrm{s} \gamma \\
-\mathrm{s} \beta & \mathrm{c} \beta \cdot \mathrm{s} \gamma & \mathrm{c} \beta \cdot \mathrm{c} \gamma
\end{array}\right]
$$

where $\mathrm{c} \alpha=\cos \alpha ; \mathrm{s} \alpha=\sin \alpha ; \quad \mathrm{c} \beta=\cos \beta ; \mathrm{s} \beta=\sin \beta ; \quad \mathrm{c} \gamma=\cos \gamma ;$ $\mathrm{s} \gamma=\sin \gamma$.

Raising the vector equation to the second power (3) and taking into consideration that $\stackrel{\mathrm{r}}{e}^{2}=1$, the following equation 
in a scalar form is obtained:

$$
\begin{aligned}
& {\left[c \alpha \cdot c \beta \cdot r_{i x}^{P}+(s \gamma \cdot s \beta \cdot c \alpha-s \alpha \cdot c \gamma) \cdot r_{i y}^{P}+X_{P}-R_{i x}\right]^{2}+} \\
& +\left[c \beta \cdot s \alpha \cdot r_{i x}^{P}+(s \alpha \cdot s \beta \cdot s \gamma+c \alpha \cdot c \gamma) \cdot r_{i y}^{P}+Y_{P}-R_{i y}\right]^{2}+ \\
& +\left(-s \beta \cdot r_{i x}^{P}+s \gamma \cdot c \beta \cdot r_{i y}^{P}+Z_{P}\right)^{2}-L_{i}^{2}=0 .
\end{aligned}
$$

To work out the mathematical model, it is convenient to present (7) in the type

$$
\begin{aligned}
& \sqrt{\left[c \alpha \cdot c \beta \cdot r_{i x}^{P}+(s \gamma \cdot s \beta \cdot c \alpha-s \alpha \cdot c \gamma) \cdot r_{i y}^{P}+X_{P}-R_{i x}\right]^{2}+} \\
& \overline{+\left[c \beta \cdot s \alpha \cdot r_{i x}^{P}+(s \alpha \cdot s \beta \cdot s \gamma+c \alpha \cdot c \gamma) \cdot r_{i y}^{P}+Y_{P}-R_{i y}\right]^{2}+} \\
& \overline{+\left(-s \beta \cdot r_{i x}^{P}+s \gamma \cdot c \beta \cdot r_{i y}^{P}+Z_{P}\right)^{2}}-L_{i}=0 \text {. }
\end{aligned}
$$

The left sides of the equations (8) are a system of functions $U_{i}\left(\vec{R}_{P}\right), U_{i}\left(\vec{R}_{P}\right)=0, i=1,2, \ldots, 6$, of the vector argument $\vec{R}_{P}=\left[\begin{array}{lllllll}X_{P} & Y_{P} & Z_{P} & \alpha & \beta & \gamma\end{array}\right]^{T}$. This function can be defined as a vector function $\vec{Q}\left(\vec{R}_{P}\right)$ of the same argument as well, i.e.

$$
\begin{aligned}
\vec{Q}\left(\vec{R}_{p}\right)= & {\left[\vec{U}_{l}\left(\vec{R}_{p}\right) \vec{U}_{2}\left(\vec{R}_{p}\right) \vec{U}_{3}\left(\vec{R}_{p}\right)\right.} \\
& \left.\vec{U}_{4}\left(\vec{R}_{p}\right) \vec{U}_{5}\left(\vec{R}_{p}\right) \vec{U}_{6}\left(\vec{R}_{p}\right)\right]^{T} .
\end{aligned}
$$

Then the Jacobian matrix for the system of equations will be:

$$
J=\frac{\partial \vec{Q}\left(\vec{R}_{P}\right)}{\partial \vec{R}_{P}^{T}}=\left[\begin{array}{llllll}
\frac{\partial U_{1}}{\partial X_{P}} & \frac{\partial U_{1}}{\partial Y_{P}} & \frac{\partial U_{1}}{\partial Z_{P}} & \frac{\partial U_{1}}{\partial \alpha} & \frac{\partial U_{1}}{\partial \beta} & \frac{\partial U_{1}}{\partial \gamma} \\
\frac{\partial U_{2}}{\partial X_{P}} & \frac{\partial U_{2}}{\partial Y_{P}} & \frac{\partial U_{2}}{\partial Z_{P}} & \frac{\partial U_{2}}{\partial \alpha} & \frac{\partial U_{2}}{\partial \beta} & \frac{\partial U_{2}}{\partial \gamma} \\
\frac{\partial U_{3}}{\partial X_{P}} & \frac{\partial U_{3}}{\partial Y_{P}} & \frac{\partial U_{3}}{\partial Z_{P}} & \frac{\partial U_{3}}{\partial \alpha} & \frac{\partial U_{3}}{\partial \beta} & \frac{\partial U_{3}}{\partial \gamma} \\
\frac{\partial U_{4}}{\partial X_{P}} & \frac{\partial U_{4}}{\partial Y_{P}} & \frac{\partial U_{4}}{\partial Z_{P}} & \frac{\partial U_{4}}{\partial \alpha} & \frac{\partial U_{4}}{\partial \beta} & \frac{\partial U_{4}}{\partial \gamma} \\
\frac{\partial U_{5}}{\partial X_{P}} & \frac{\partial U_{5}}{\partial Y_{P}} & \frac{\partial U_{5}}{\partial Z_{P}} & \frac{\partial U_{5}}{\partial \alpha} & \frac{\partial U_{5}}{\partial \beta} & \frac{\partial U_{5}}{\partial \gamma} \\
\frac{\partial U_{6}}{\partial X_{P}} & \frac{\partial U_{6}}{\partial Y_{P}} & \frac{\partial U_{6}}{\partial Z_{P}} & \frac{\partial U_{6}}{\partial \alpha} & \frac{\partial U_{6}}{\partial \beta} & \frac{\partial U_{6}}{\partial \gamma}
\end{array}\right] .
$$

The system of scalar equations (8) and the Jacobian matrix entirely determine the geometric kinematics of the 6jack Stewart platform.

However, on the other hand, the system of equations (8) is non-linear. Its solutions, which determine the motion coordinates of the operating platform, should be sought by numerical methods [14]. One of these methods is the Newton-Rafson method. According to it the vector of the output coordinates is defined by the iterative formula [15, $16]$

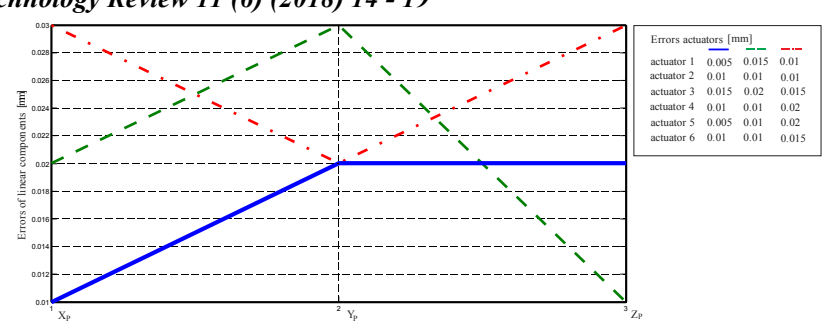

Fig. 5. Errors of the linear components of vector $\vec{R}_{P}\left[\begin{array}{lll}\mathrm{X}_{\mathrm{P}} & \mathrm{Y}_{\mathrm{P}} & \mathrm{Z}_{\mathrm{P}}\end{array}\right]$

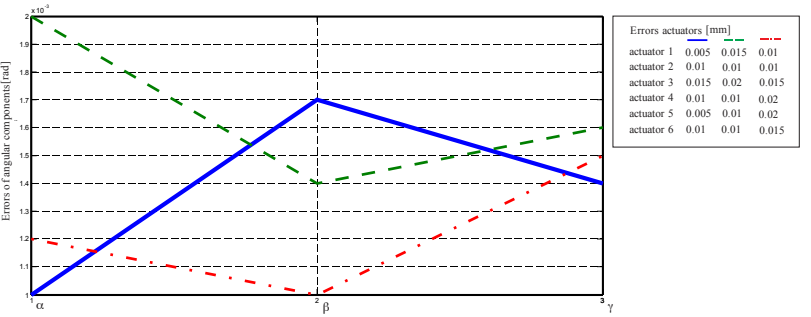

Fig. 6. Errors of the angular components of vector $\vec{R}_{P}\left[\begin{array}{lll}\alpha \beta & \beta\end{array}\right]$

$$
\left(\vec{R}_{P}\right)_{j+1}=\left(\vec{R}_{P}\right)_{j}-\left[\frac{\partial \vec{Q}\left(\vec{R}_{P}\right)_{j}}{\partial\left(\vec{R}_{P}^{T}\right)_{j}}\right]^{-1} \cdot \vec{Q}\left(\vec{R}_{P}\right)_{j}
$$

In is assumed in (11) that the initial configuration of the platform is known. For each of the calculation steps the values of the components of the vector $\vec{R}_{P}$, obtained for the previous step, are accepted as initial approximations.

This procedure can be used for defining the error of the operating platform upon its motion according to a set trajectory. Since the latter is determined by vector $\vec{R}_{P}$, then it follows that its errors defined by vector $\Delta \vec{R}_{P}=\left[\begin{array}{llllll}\Delta X_{P} & \Delta Y_{P} & \Delta Z_{P} & \Delta \alpha & \Delta \beta & \Delta \gamma\end{array}\right]$ are functions of the errors identified in the lengths of the actuators and set in (11) by the components of vector $\Delta \vec{L}=\left[\begin{array}{llllll}\Delta L_{1} & \Delta L_{2} & \Delta L_{3} & \Delta L_{4} & \Delta L_{5} & \Delta L_{6}\end{array}\right]$. To present the components of vector $\Delta \vec{L}$ in a clear form in the iterative formula (11), we designate the quantity under the radical sign from (8) with $\left\|a_{i}^{w}\right\|, i=1,2, \ldots, 6$. Then taking into account all the above mentioned specifications, we obtain the following final formula defining the procedure for determining the accuracy of motion of the motion platform according to the set trajectory.

$$
\left[\begin{array}{c}
\Delta X_{P} \\
\Delta Y_{P} \\
\Delta Z_{P} \\
\Delta \alpha \\
\Delta \beta \\
\Delta \gamma
\end{array}\right]_{j+1}=\left[\begin{array}{c}
X_{P} \\
Y_{P} \\
Z_{P} \\
\alpha \\
\beta \\
\gamma
\end{array}\right]_{j}\left[\begin{array}{l}
\left\|J^{w}\right\|-\Delta L_{1} \\
\left\|a_{2}^{w}\right\|-\Delta L_{2} \\
\left\|a_{3}^{w}\right\|-\Delta L_{3} \\
\left\|a_{4}^{w}\right\|-\Delta L_{4} \\
\left\|a_{5}^{w}\right\|-\Delta L_{5} \\
\left\|a_{6}^{w}\right\|-\Delta L_{6}
\end{array}\right] .
$$


The iterative formula (12) for each initial platform configuration selected should result in a unique solution. To satisfy this condition, it is required that the determinant of the matrix (10) is not equal to zero for all possible values of vector $\stackrel{L}{P}_{P}$, forming upon the implementation of the set law of motion of the operating platform, i.e.

$$
\operatorname{det} J=\operatorname{det} \frac{\partial \vec{Q}\left(\vec{R}_{P}\right)}{\partial \vec{R}_{P}^{T}} \neq 0[17,18,19]
$$

The above described mathematical model, output measuring instrument and hierarchical diagram establish all required conditions that form reference qualities in measuring equipment. A significant advantage of the hierarchical diagram is that the output measuring tool is calibrated in static mode.

The software implementation of the mathematical model determining indirectly the accuracy of reproduction of the set motions of the operating platform is realized in Matlab. The results obtained from the investigation of the accuracy of the measuring equipment are visualized in fig. 5 and 6 . The two figures illustrate the errors of, respectively, the linear and angular components of the vector defining the trajectory of the operating platform. The investigation is realized on the basis of three groups of experimental results obtained upon the accuracy analysis of each actuator for three different weight loads.

\section{Conclusions}

The above mathematical models and the output measuring device make possible the development of metrologically traceable calibration hierarchy from a measurement standard of a static quantity to equipment with reference qualities in dynamic measurement mode. This enables us to link the measurement result of dynamic quantities to a reference of a static quantity. In this case the reference of the static quantity is the definition of a measurement unit of length through its practical realization.

In addition, by means of the proposed mathematical model, the referent measuring instrument and the hierarchical diagram the measuring equipment can not only be calibrated and verified but also its accuracy can be modeled at the stage of its development.

This is due to the fact that the summarized mathematical model defines the positional input-output relation between the lengths of the actuators and the output coordinates of the motion platform. Taking into consideration that the actuators are the most important design elements in terms of the accuracy and dynamics of the Stewart platform, the efficiency of the proposed approach can be seen. The results from the experimental tests confirm the reliability of the mathematical model developed.

\section{Acknowledgements}

This work was supported by the European Regional Development Fund within the Operational Programme "Science and Education for Smart Growth 2014 - 2020" under the Project $\mathrm{CoE}$ "National center of mechatronics and clean technologies“ BG05M2OP001-1.001-0008-C01.

This is an Open Access article distributed under the terms of the Creative Commons Attribution License

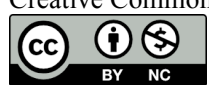

\section{References}

[1] Jiang, M., L. Jiang, D. Jiang, J. Xiong, J. Shen, S. H. Ahmed, J. Luo, H. Song. Dynamic measurement errors prediction for sensors based on firefly algorithm optimize support vector machine. Sustainable Cities and Society, 35, 2017, 250-256, ISSN 2210-6707.

[2] Jiang, M., J. Luo, D. Jiang, J. Xiong, H. Song, J. Shen. A cuckoo search-support vector machine model for predicting dynamic measurement errors of sensors. IEEE Access, 4, 2016, 5030-5037, ISSN 2169-3536.

[3] Dichev, D., H. Koev, T. Bakalova, P. Louda. A Model of the Dynamic Error as a Measurement Result of Instruments Defining the Parameters of Moving Objects. Measurement Science Review, 14 (4), 2014, 183-189, ISSN 1335-8871.

[4] Kostadinova, L., Shopov, N., Madjarov, N., Jelezarov, I. New Computer-Aided Ultrasonic Technology for Classification of Factory Produced Bulgarian Yogurt. Comptes Rendus de l'Academie Bulgare des Sciences, 68(1), 2015, 89-94, ISSN 13101331.

[5] Málek, V., Makowka, M., Kormunda, M., Ryšánek, P. Tribological properties and abrasion resistance thin films of tungsten carbide. METAL 2017 - 26th International Conference on Metallurgy and Materials, Brno, Szech Republic, 2017, pp. 1372 - 1376, ISBN 978-80-87294-79-6.

[6] Nilsson, J-O., I. Skog. Inertial Sensor Arrays - A Literature Review. 24th European Navigation Conference, IEEE, Finland, 2016, ISBN 978-147998915-7.

[7] Dichev, D., H. Koev, D. Diakov, N. Panchev, R. Miteva, H. Nikolova. Automated System for Calibrating Instruments Measuring Parameters of Moving Objects. 59th International Symposium ELMAR, September 18-20th, 2017, Zadar, Croatia, 219-224, ISSN 1334-2630.

[8] Fichter, E. F. A Stewart Platform-Based Manipulator: General Theory and Practical Construction. Int. Journ. Robot. Res., 5 (2), 1986, 157-182, ISSN: 0278-3649.
[9] Raghavan, M. The Stewart Platform of General Geometry Has 40 Configurations. Journ. Mech. Des., 115(2), 1993, 277-232, ISSN 1050-0472.

[10] Dasgupta, B. A canonical formulation of the direct position kinematics problems for a general 6-6 Stewart platform. Mecnanism and Machine Theory, 29(6), 1994, 819-827, ISSN 0094-114X.

[11] Lee, T. Y., Shim, J. K. Forward kinematics of the general 6-6 Stewart platform using algebraic elimination. Mechanism and Machine Theory, 36(9), 2001, 1073-1085, ISSN: 0094-114X.

[12] Huang, X., Liao, Q., Wei, S. Closed-form forward kinematics for a symmetrical 6-6 Stewart platform using algebraic elimination. Mechanism and Machine Theory, 45(2), 2010, 327-334, ISSN: 0094-114X.

[13] Dichev, D., H. Hasanov, H. Koev. Mathematical Modelling of the Kinematics of Stand Equipment for Investigating the Accuracy Characteristics of Instruments Measuring the Psarameters of Moving Objects. Mechanics of Machines Magazine, TU-Varna, Book 2, 2013.

[14] Chen, S. H., Fu, L. C. Output feedback sliding mode control for a Stewart platform with a nonlinear observer-based forward kinematics solution. IEEE Transactions on control systems technology, 21(1), 2013, 176-185, ISSN: 1063-6536.

[15] Dichev, D., F. Kogia, H. Koev, D. Diakov. Method of Analysis and correction of the Error from Nonlinearity of the Measurement Instruments. Journal of Engineering Science and Technology Review. 9(6), 2016, 116-121, ISSN 1791-2377.

[16] Malakov, I., V. Zaharinov, G. Dinev. Decision Making Support System for Multicriteria Discrete Optimization of Technical Products. Applied Mechanics \& Materials. Vol. 841, 2016, 53-58, ISSN: 1662-7482.

[17] Su, Y. X., Duan, B. Y., Zheng, C. H., Zhang, Y. F., Chen, G. D., Mi, J. W. Disturbance-rejection high-precision motion control of a 
Dimitar Dichev, Fotini Kogia, Hristiyana Nikolova and Dimitar Diakov/

Journal of Engineering Science and Technology Review 11 (6) (2018) 14 - 19

Stewart platform. IEEE transactions on control systems technology,

12(3), 2004, 364-374, ISSN: 1063-6536.

[18] Dymarek, A., Dzitkowski, T., Herbuś, K., Kost, G., Ociepka, P. ().

Geometric analysis of motions exercised by the Stewart platform. Advanced Materials Research, vol. 837, 2014, 351-356, ISSN

\section{$1662-8985$.}

[19] Hinkov, A., M. Milushev. Decentraized Control of Intralogistics Order Picking Systems with Can. 30th International Conference on Information Technologies - InfoTech'2016, 291-300, ISSN 13141023. 